\title{
Serum PTH associated with malnutrition determined by bioelectrical impedance technology in chronic kidney disease patients
}

Lilin Liu

Center for Kidney Disease, Second Affiliated Hospital of Nanjing Medical University

\section{Lulu Wang}

Center for Kidney Disease, Second Affiliated Hospital of Nanjing Medical University

\section{Xiao Wang}

Center for Kidney Disease, Second Affiliated Hospital of Nanjing Medical University

\section{Mingxia Xiong}

Center for Kidney Disease, Second Affiliated Hospital of Nanjing Medical University

\section{Hongdi Cao}

Center for Kidney Disease, Second Affiliated Hospital of Nanjing Medical University

\section{Lei Jiang}

Center for Kidney Disease, Second Affiliated Hospital of Nanjing Medical University

Junwei Yang ( $\sim$ jwyang@njmu.edu.cn )

Center for Kidney Disease, Second Affiliated Hospital of Nanjing Medical University

\section{Research Article}

Keywords: PTH, malnutrition, bioelectrical impedance technology, chronic kidney disease

Posted Date: June 9th, 2021

DOl: https://doi.org/10.21203/rs.3.rs-596127/v1

License: (c) (1) This work is licensed under a Creative Commons Attribution 4.0 International License.

Read Full License 


\section{Serum PTH associated with malnutrition determined by bioelectrical impedance}

technology in chronic kidney disease patients

Lilin Liü, Lulu Wang", Xiao Wang, Mingxia Xiong, Hongdi Cao*, Lei Jiang*, Junwei Yang*

${ }^{1}$ Center for Kidney Disease, 2nd Affiliated Hospital, Nanjing Medical University, Nanjing, Jiangsu 210003, China

Running title: PTH and malnutrition in CKD patients

\# Lilin Liu and Lulu Wang contributed equally to this work.

*The Correspondences:

Dr. Junwei Yang: Center for Kidney Disease, Second Affiliated Hospital of Nanjing Medical University; 262\# North Zhongshan Road, Nanjing, 210003, China. Email: jwyang@njmu.edu.cn;

Dr. Lei Jiang: Center for Kidney Disease, Second Affiliated Hospital of Nanjing Medical University; 262\# North Zhongshan Road, Nanjing, 210003, China. Email: jianglei@njmu.edu.cn

Dr. Hongdi Cao: Center for Kidney Disease, Second Affiliated Hospital of Nanjing Medical University; 262\# North Zhongshan Road, Nanjing, 210003, China. Email: caohongdi@njmu.edu.cn 


\begin{abstract}
Background: Chronic malnutrition and cachexia are common in chronic kidney disease (CKD), and importance should be given to these complications because they affect the patient's quality of life and the prognosis. This sudy analyze the correlation between serum PTH level and nutritional status and body composition of patients with CKD.

Methods: CKD patients were enrolled in Center for Kidney Disease, Second Affiliated Hospital of Nanjing Medical University from December 1, 2016 to November 30, 2020. Bioelectrical impedance technology was applied to examine body composition. The characteristics of the body composition were compared among different stage of CKD patients, then the correlation between PTH and the body composition was analyzed.
\end{abstract}

Results: 205 CKD patients were enrolled. Twenty-five patients were in stage 1 or 2 CKD, 78 patients were in stage 3 or 4,31 patients were in stage 5 without dialysis (referred to as CKD stage 5A), and 71 patients were in stage 5 with dialysis (referred to as CKD stage $5 \mathrm{~B}$ ). Body composition analysis showed that the patients had a phase angle (PA) of $5.02 \pm 1.07^{\circ}$, a percentage of body fat $(\mathrm{PBF})$ of $27.74 \pm 8.8 \%$, and a skeletal muscle mass index (SMI) of $7.4 \pm 1.34 \mathrm{~kg} / \mathrm{m}^{2}$. PBF peaked in the CKD stage 3/4 group and gradually decreased with the progression of CKD. PA and SMI differed significantly between the CKD stage $1 / 2$ and stage $5 \mathrm{~B}$ groups. The proportion of low SMI did not differ significantly between the CKD stage $1 / 2$ and stage $3 / 4$ groups, but it was obviously higher in the CKD stage 5A and 5B groups. PTH was significantly correlated with BMI, hemoglobin, albumin, total cholesterol, triglycerides, and SMI .Binary logistic regression of low SMI showed that the odds ratio for PTH levels greater than the upper limit of the normal range was $11.769(\mathrm{p}=0.043,95 \%$ 
confidence interval: $1.078-128.536$ ), and the model predictive power was 0.986 after correction for age, sex, height, weight, hemoglobin, serum calcium, serum phosphorus, serum total cholesterol, serum triglyceride, and basal metabolic rate.

Conclusion: Bioelectrical impedance can effectively assess the nutritional status of CKD patients in terms of fat and muscle parameters. High levels of PTH are an independent risk factor for developing low SMI in CKD patients.

Keywords: PTH, malnutrition, bioelectrical impedance technology, chronic kidney disease 


\section{Introduction}

Chronic kidney disease (CKD) is a global health problem, and its prevalence is increasing every year due to the change in disease spectrum. Chronic malnutrition and cachexia are common in CKD patients, and importance should be given to these complications because they affect the patient's quality of life and may even be associated with depression[1]. Severe protein malnutrition significantly increases the mortality of CKD patients[2]. In contrast to malnutrition due to other causes, CKD patients often develop an imbalance between energy requirement and nutritional intake leading to loss of protein and fat mass, which cannot be readily corrected by dietary supplementation regimens [3] .

Previous physiological studies[4, 5] have shown that parathyroid hormone $(\mathrm{PTH}) / \mathrm{PTH}-$ related protein $(\mathrm{PTHrP})$ induces browning of white fat, increases basal metabolic rate, and affects muscle mass and have verified the upregulation of thermogenic gene transcription in patients with primary hyperparathyroidism. CKD patients also have high levels of circulating PTH. However, the relationship between PTH and the nutritional status and body composition of CKD patients remains unknown. Traditional nutritional indicators (e.g., albumin, prealbumin, cholesterol, etc.) are generally used to assess the overall nutritional status, while body composition analyzers can provide a wealth of information regarding the basal metabolic rate and the mass and distribution of muscle and fat through bioelectrical impedance technology[6].

Therefore, the purpose of this study was to analyze the nutritional status and body composition of CKD patients using bioelectrical impedance technology, investigate the differences in nutritional status among patients at different stages of $\mathrm{CKD}$, and analyze the correlation between PTH levels and body composition of CKD patients. 


\section{Methods}

\section{Study population and setting}

The participants were enrolled from hospitalized patients in Center for Kidney Disease, Second Affiliated Hospital of Nanjing Medical University from December 1, 2016 to November 30, 2020. The inclusion criteria included aged above 18 years old and diagnosed as CKD according to KDIGO guidelines[7]. The exclusion criteria included: (1) acute infection, (2) life expectancy of less than 1 year due to decompensated liver cirrhosis or malignant tumor, and (3) malignant hypertension ( $\mathrm{SBP} \geq 180 \mathrm{mmHg}$ or $\mathrm{DBP} \geq 110 \mathrm{mmHg}$ with clinical symptoms). All participants signed the informed consent, and this study was certificated and approved by the Ethics Committee of Second Affiliated Hospital of Nanjing Medical University (Document Number: [2015] KY052).

\section{Bioelectrical impedance analysis of body composition}

After seated for at least 20 minutes, the participants were examined for human body composition using the biological resistance technology (Korea; Inbody S10). Water drinking was forbidden 2 hours before the examination. Hemodialysis patients were examined half an hour after hemodialysis. Based on the nutritional content evaluated by Inbody, the bioelectrical impedance results were categorized into three types of nutritional indicators. Total nutritional indicators included: phase angle (PA), body cell mass $(\mathrm{BCM})$, protein, basal metabolic rate (BMR); fat indicators included: percent body fat (PBF), visceral fat area (VFA), body fat mass (BFM); muscle indicators included: fat free mass (FFM), soft lean mass (SLM), skeletal muscle mass (SMM), skeletal muscle index (SMI). Here, low PA was defined as less than $5^{\circ}$ in men and less than $4.6^{\circ}$ in women[8], and low SMI was defined as less than $7 \mathrm{~kg} / \mathrm{m}^{2}$ in men and less than $5.7 \mathrm{~kg} / \mathrm{m}^{2}$ in women[9]. 


\section{General materials and laboratory tests}

The demographic and medical information of all subjects were collected. Fasting blood was sampled on the morning of the same day for routine laboratory examination, including hemoglobin, serum albumin, serum total cholesterol, serum triglyceride, serum urea nitrogen, serum creatinine, serum phosphorus, serum calcium and plasma PTH. PTH was detected by chemiluminescence immunoassay (America; BECKMANDx1800 immunoassay system), and the upper limit of the normal detection range, $88 \mathrm{mmol} / \mathrm{L}$, was used as the cut-off value.

\section{Statistical analyses}

The data of normal distribution were described in the form of mean \pm standard deviation, the data of non-normal distribution were described in the form of median, and the classified variables were described in the form of frequency (percentage). T-test was used to compare the continuous variables of normal distribution between two groups, one-way ANOVA was used to compare among three or more groups, and nonparametric test was used to compare the continuous variables of non-normal distribution. Chi-square test was used to compare the rates. The correlation between variables was analyzed by Pearson correlation analysis, in which the non-normal distribution data was transformed into normal distribution data by natural logarithm. Binary logistic regression was used to explore the risk factors of low SMI. All analyses were performed with SPSS 26.0. It is statistically significant when the $P$ value $<0.05$.

\section{Results}

\section{Basic information}

This study enrolled 205 CKD patients, who were divided into groups based on the CKD stage. Twenty-five patients were in stage 1 or 2 CKD, 78 were in stage 3 or 4 , 
31 were in stage 5 without dialysis (referred to as CKD stage 5A), and 71 were in stage 5 with dialysis (referred to as CKD stage 5B). Sixty-two percent of the patients was male with median age 56 years old. Diabetic nephropathy was the most common etiology of CKD (30.7\%) among the patients, followed by glomerulonephropathy (24.9\%). As shown in Table 1, there were statistical differences in age, body mass index (BMI) and etiology among groups while no statistical differences in sex ratio.

\section{Differences in nutritional indicators among patients in different CKD stages}

As summarized in Table 1, CKD patients had a mean BMI of $24.93 \mathrm{~kg} / \mathrm{m}^{2}$, a median serum albumin level of $39.7 \mathrm{~g} / \mathrm{L}$, a median serum total cholesterol level of 4.14 $\mathrm{mmol} / \mathrm{L}$, and a median serum triglyceride level of $1.69 \mathrm{mmol} / \mathrm{L}$. As summarized in Table 2, body composition analysis showed that the patients had a phase angle (PA) of $5.02 \pm 1.07^{\circ}$, a percentage of body fat (PBF) of $27.74 \pm 8.8 \%$, and a skeletal muscle mass index (SMI) of $7.4 \pm 1.34 \mathrm{~kg} / \mathrm{m}^{2}$. There were significant differences in the levels of overall nutritional and muscle indicators among the four groups except for serum albumin level and proportion of patients with low PA. Apart from body fat mass (BFM), there were no significant differences in body fat indicators among the groups.

With the progression of CKD, BMI, total cholesterol, and triglycerides decreased gradually. Albumin level varied greatly among the patients in the CKD stage $1 / 2$ group, but the level increased in the other three groups with the progression of CKD. A significant difference was present only between the groups of CKD stage $3 / 4$ and stage 5B. PBF peaked in the patients of CKD stage 3/4 and gradually decreased with the progression of CKD. PA and SMI differed significantly between the groups of CKD stage $1 / 2$ and $5 \mathrm{~B}$, but not between the groups of CKD stage $3 / 4$ and $5 \mathrm{~A}$. Further analysis (Figure 1) revealed that the proportion of low SMI did not differ significantly between the the groups of CKD stage $1 / 2$ and $3 / 4$, but it was obviously higher in the 
groups of CKD stage 5A and 5B.

\section{Correlation analysis of PTH and nutritional indicators}

The mean PTH level of the patients was $103.5 \mathrm{pg} / \mathrm{ml}$ at the time of enrollment, and it showed marked increase with the progression of CKD. PTH was significantly correlated with BMI, hemoglobin, albumin, total cholesterol, triglycerides, and SMI but not with PA, PBF, and BMR (Table 3).

\section{Risk factors for low SMI}

The low-SMI group was compared with the normal SMI group (Table 4). Binary logistic regression of low SMI (Table 5) showed that the odds ratio for PTH levels greater than the upper limit $(88 \mathrm{pg} / \mathrm{ml})$ of the normal range was $11.769(\mathrm{p}=0.043,95 \%$ confidence interval: $1.078-128.536$ ), and the model predictive power was 0.986 (Table 5) after correction for age, sex, height, weight, hemoglobin, serum calcium, serum phosphorus, serum total cholesterol, serum triglyceride, and basal metabolic rate.

\section{Discussion}

The nutritional status of patients gradually declined with the progression of CKD, and the changes in body composition manifested mainly as the decrease in SMI, while the changes in body fat indicators were not considerable. The proportion of hemodialysis patients with low PA and SMI was significantly higher than that of non-dialysis patients at any CKD stage. High levels of PTH were an independent risk factor for developing low SMI in CKD patients.

CKD patients often develop a unique nutritional imbalance, resulting from increase in energy requirements (caused by catabolism and chronic inflammation) with paradoxical reduction in appetite, for which the International Society of Renal Nutrition and Metabolism proposed the concept of protein-energy wasting (PEW) in 
2008[10]. PEW is among the strongest predictors of mortality in CKD patients (hazard ratio 3.03) [11]. Diagnosis and treatment of PEW is a key step in the clinical nutritional and overall management of CKD. At present, the diagnosis of PEW is established on the basis of changes in serum biochemical indices, BMI, muscle mass, and dietary intake [10]. Traditional methods for evaluating muscle mass and judging changes in food intake lack uniformity, interrater reliability, and accuracy; while dynamic changes in the water load of hemodialysis patients greatly impact the body weight. On the other hand, assessment of skeletal muscle mass for all CKD patients through radionuclide imaging[9] results in excessive economic burden on the healthcare system. Bioelectrical impedance technology was adopted in this study to assess the characteristics and changes in body composition of CKD patients. This technology is non-invasive, convenient, and cost-efficient. It can be effectively employed in lieu of the dual-energy X-ray method[12] and metabolic carts[13] to assess objectively and reliably the amount of water, muscle, and fat mass present in the bodies of CKD patients as well as their basal metabolic rate, which reflect the nutritional status of these patients, thereby having an increased clinical value.

High PTH level is an important risk factor for poor prognosis in CKD patients. During the development and progression of $\mathrm{CKD}$, PTH not only causes bone demineralization but also affects the proper functioning of the brain, heart, and the immune system[14]. This clinical analysis revealed that the PTH was associated with the nutritional status, especially the muscle mass, of CKD patients. The proportion of patients with low SMI was high in the group of CKD stage 5B, while further analysis revealed relatively normal or even slightly high levels of hemoglobin, albumin, and serum phosphorus in these patients, thereby indicating adequate nutritional intake. However, prevalence of sarcopenia is significantly higher in patients undergoing 
hemodialysis due to accelerated protein catabolism despite adequate nutrition [15]. Good muscle function is one of the key factors that aids CKD patients in resuming their daily activities. Considering that dietary interventions alone may not improve the nutritional status and muscle function of hemodialysis patients, it is necessary to diagnose malnutrition and PEW early so that appropriate treatment can be provided. The effect of PTH on muscle tissue is not specific to CKD patients, and similar phenomena have been observed in experimental models of tumors $[4,5]$ in patients with primary hyperparathyroidism [4], in female patients with vitamin D deficiency[16], and in the elderly population[17], while this association has not been observed among the general population[18]. From a physiological perspective, PTH/PTHrP activates protein kinase A and thermogenic genes such as uncoupling protein 1 (Ucp1), induces browning of white adipocytes, increases basal metabolic rate, and mediates energy wasting, which in turn leads to muscular atrophy and a decline in muscle function $[4,19]$. At the cellular level, this manifests as a decrease in both mitochondrial activity and high-energy phosphate content in muscle tissue[20]. The present study provides clinical evidence regarding the effect of high PTH levels on muscle tissue, which may direct future research into the treatment of sarcopenia in CKD patients.

In the present study, albumin levels first increased and then decreased with the progression of CKD, which was different from the trend for most nutritional indicators. The discrepancy may be attributed to the fact that the underlying conditions leading to $\mathrm{CKD}$ mainly included diabetic nephropathy and primary glomerulonephritis, which frequently cause proteinuria. Degree of proteinuria correlates with the patient's renal function outcomes [21], which explains the high degree of proteinuria in CKD patients before dialysis. In contrast, as residual renal 
function is lost when dialysis is started, urine output gradually decreases and the patient's dietary restrictions become fewer than before, allowing for the rise and maintenance of serum albumin levels. Therefore, CKD stage 3/4 patients exhibited the lowest mean level of serum albumin in this study.

There are some limitations in this study. First of all, this was a cross-sectional study and, thus, unable to reveal causality. Further research is needed to confirm whether intervention to reduce PTH levels can serve as an effective treatment for malnutrition and PEW in CKD patients. In view of the U-shaped relationship curve between the PTH level and the mortality risk of CKD patients [22], it is not clear whether simple parathyroidectomy will be able to improve the short- and long-term prognosis of patients. Moreover, the optimal target range for PTH level that should be achieved by using calcimimetics and activated vitamin $\mathrm{D}$ in order to reduce the risk of developing low SMI is not known. These issues need to be resolved through long-term follow-up. Secondly, unlike previous physiological research, this study did not observe a correlation of PTH levels with body fat indicators (except for serum total cholesterol) and basal metabolic rate. However, this finding does not necessarily confirm the absence of such correlation. For ethical reasons, it is not possible to obtain adipose tissue from deeper sites from patients to determine whether the expression of thermogenic genes is up-regulated. Therefore, the lack of observable correlation may be interpreted as arising from the possible transient effects of PTH on fats and basal metabolic rate as well as the relatively small sample size in this study. A larger sample size is needed to further verify the above observation and postulation. In addition, the self-controlled comparison of patients after parathyroidectomy may provide some evidence for the effects of PTH on fats and basal metabolic rate. Therefore, subsequent clinical research should focus on the establishment of a 
reasonable control range for $\mathrm{PTH}$ and the observation of post-parathyroidectomy changes in patient's body composition.

\title{
Conclusions
}

In summary, bioelectrical impedance can effectively assess the nutritional status of CKD patients in terms of fat and muscle parameters. High levels of PTH are an independent risk factor for developing low SMI in CKD patients. It is necessary to pay more attention to the nutritional management of $\mathrm{CKD}$ patients and the remodeling of fat and muscle distribution. Moreover, PTH may be a potential therapeutic target for treating sarcopenia in CKD patients.

\begin{abstract}
Abbreviations
CKD, Chronic kidney disease; IQR, interquartile range; $\mathrm{PTH}$, parathyroid hormone; $\mathrm{Hb}$, hemoglobin; Alb, albumin; Tc, total cholesterol; Tg, triglyceride; PA, phase angle; BMR, basal metabolic rate; PBF, percent body fat; SMI, skeletal muscle index.
\end{abstract}

\section{Acknowledgments}

We thank the participating patients and the medical staff in Center for Kidney Disease, 2nd Affiliated Hospital, Nanjing Medical University for making the study possible.

\section{Authors' contributions}

J Y, L J and H C contributed to the study conception and design. Material preparation, data collection and analysis were performed by L L, and L W. The first draft of the manuscript was written by $\mathrm{L} \mathrm{J}$ and all authors commented on previous versions of the manuscript. All authors read and approved the final manuscript.

\section{Funding}

This works was supported by National Natural Science Foundation of China Grants 81870502 and Jiangsu Province's Key Provincial Talents Program:Qnrc2016669 to Lei Jiang; National Natural Science Foundation of China Grants 81873618, the Key project of National Natural Science Foundation of China 81530022, and Science and Technology Support Program of Jiangsu Province: BE2017762 to Junwei Yang; National Science Foundation of China Grants81800653 
and 82070761 to Hongdi Cao.

\section{Availability of data and materials}

The datasets used and/or analysed during the current study are available from the corresponding author on reasonable request.

\section{Declarations}

\section{Ethics approval and consent to participate}

The study complies with the declaration of Helsinki. All participants signed the informed consent, and this study was certificated and approved by the Ethics Committee of Nanjing Medical University's Second Affiliated Hospital (document number: [2015] KY052).

\section{Consent for publication}

Not applicable.

\section{Competing interests}

The authors declare that they have no competing interests.

\section{Reference:}

1. Ibrahim S, El Salamony O: Depression, quality of life and malnutrition-inflammation scores in hemodialysis patients. Am J Nephrol 2008, 28(5):784-791.

2. Zha Y, Qian Q: Protein Nutrition and Malnutrition in CKD and ESRD. Nutrients 2017, 9(3).

3. Wang XH, Mitch WE: Mechanisms of muscle wasting in chronic kidney disease. Nat Rev Nephrol 2014, 10(9):504-516.

4. Kir S, Komaba H, Garcia AP, Economopoulos KP, Liu W, Lanske B, Hodin RA, Spiegelman BM: PTH/PTHrP Receptor Mediates Cachexia in Models of Kidney Failure and Cancer. Cell Metab 2016, 23(2):315-323.

5. Kir S, White JP, Kleiner S, Kazak L, Cohen P, Baracos VE, Spiegelman BM: Tumour-derived PTH-related protein triggers adipose tissue browning and cancer cachexia. Nature 2014, 513(7516): 100-104.

6. Ceniccola GD, Castro MG, Piovacari SMF, Horie LM, Corrêa FG, Barrere APN, Toledo DO: Current technologies in body composition assessment: advantages and disadvantages. Nutrition (Burbank, Los Angeles County, Calif) 2019, 62:25-31.

7. Stevens PE, Levin A: Evaluation and management of chronic kidney disease: synopsis of the kidney disease: improving global outcomes 2012 clinical practice guideline. Annals of internal medicine 2013, 158(11):825-830.

8. Kyle UG, Genton L, Pichard C: Low phase angle determined by bioelectrical impedance analysis is associated with malnutrition and nutritional risk at hospital admission. Clinical nutrition (Edinburgh, Scotland) 2013, 32(2):294-299.

9. Chen LK, Liu LK, Woo J, Assantachai P, Auyeung TW, Bahyah KS, Chou MY, Chen LY, Hsu PS, Krairit $\mathrm{O}$ et al: Sarcopenia in Asia: consensus report of the Asian Working Group for Sarcopenia. Journal of the American Medical Directors Association 2014, 15(2):95-101.

10. Fouque D, Kalantar-Zadeh K, Kopple J, Cano N, Chauveau P, Cuppari L, Franch H, Guarnieri G, Ikizler TA, Kaysen $G$ et al: A proposed nomenclature and diagnostic criteria for protein-energy wasting in acute and chronic kidney disease. Kidney Int 2008, 73(4):391-398.

11. Koppe L, Fouque D, Kalantar-Zadeh K: Kidney cachexia or protein-energy wasting in chronic kidney disease: facts and numbers. J Cachexia Sarcopenia Muscle 2019, 10(3):479-484.

12. McLester CN, Nickerson BS, Kliszczewicz BM, McLester JR: Reliability and Agreement of Various InBody Body Composition Analyzers as Compared to Dual-Energy X-Ray Absorptiometry in Healthy Men and Women. Journal of clinical densitometry : the official journal of the International Society for Clinical Densitometry 2020, 23(3):443-450. 
13. Salacinski AJ, Howell SM, Hill DL: Validity of the InBody $520^{\mathrm{TM}}$ to predict metabolic rate in apparently healthy adults. The Journal of sports medicine and physical fitness 2018, 58(9):1275-1280.

14. Urena-Torres PA, Vervloet M, Mazzaferro S, Oury F, Brandenburg V, Bover J, Cavalier E, Cohen-Solal M, Covic A, Drueke TB et al: Novel insights into parathyroid hormone: report of The Parathyroid Day in Chronic Kidney Disease. Clin Kidney J 2019, 12(2):269-280.

15. Sabatino A, Cuppari L, Stenvinkel P, Lindholm B, Avesani CM: Sarcopenia in chronic kidney disease: what have we learned so far? J Nephrol 2020.

16. Bislev LS, Langagergaard Rodbro L, Sikjaer T, Rejnmark L: Effects of Elevated Parathyroid Hormone Levels on Muscle Health, Postural Stability and Quality of Life in Vitamin D-Insufficient Healthy Women: A Cross-Sectional Study. Calcif Tissue Int 2019, 105(6):642-650.

17. Visser M, Deeg DJ, Lips P, Longitudinal Aging Study A: Low vitamin D and high parathyroid hormone levels as determinants of loss of muscle strength and muscle mass (sarcopenia): the Longitudinal Aging Study Amsterdam. J Clin Endocrinol Metab 2003, 88(12):5766-5772.

18. Marantes I, Achenbach SJ, Atkinson EJ, Khosla S, Melton LJ, 3rd, Amin S: Is vitamin D a determinant of muscle mass and strength? J Bone Miner Res 2011, 26(12):2860-2871.

19. Thomas SS, Mitch WE: Parathyroid hormone stimulates adipose tissue browning: a pathway to muscle wasting. Curr Opin Clin Nutr Metab Care 2017, 20(3):153-157.

20. Baczynski R, Massry SG, Magott M, el-Belbessi S, Kohan R, Brautbar N: Effect of parathyroid hormone on energy metabolism of skeletal muscle. Kidney Int 1985, 28(5):722-727.

21. Cravedi P, Ruggenenti P, Remuzzi G: Proteinuria should be used as a surrogate in CKD. Nat Rev Nephrol 2012, 8(5):301-306.

22. Floege J, Kim J, Ireland E, Chazot C, Drueke T, de Francisco A, Kronenberg F, Marcelli D, Passlick-Deetjen J, Schernthaner G et al: Serum iPTH, calcium and phosphate, and the risk of mortality in a European haemodialysis population. Nephrol Dial Transplant 2011, 26(6):1948-1955. 
Table 1. Basic indicators of different CKD stage groups

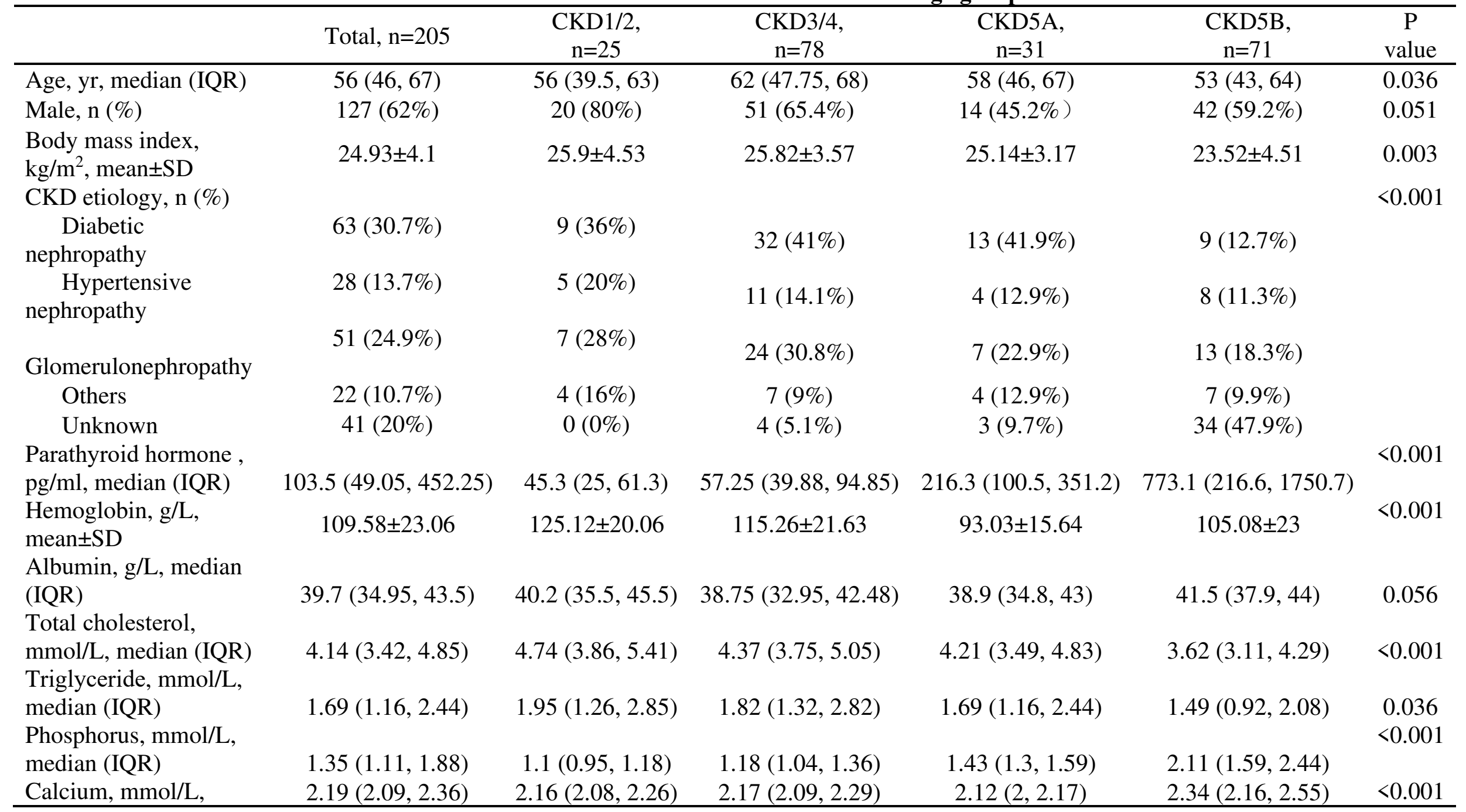


median (IQR)

IQR, interquartile range. 
Table 2. Nutritional indicators of different CKD stage groups

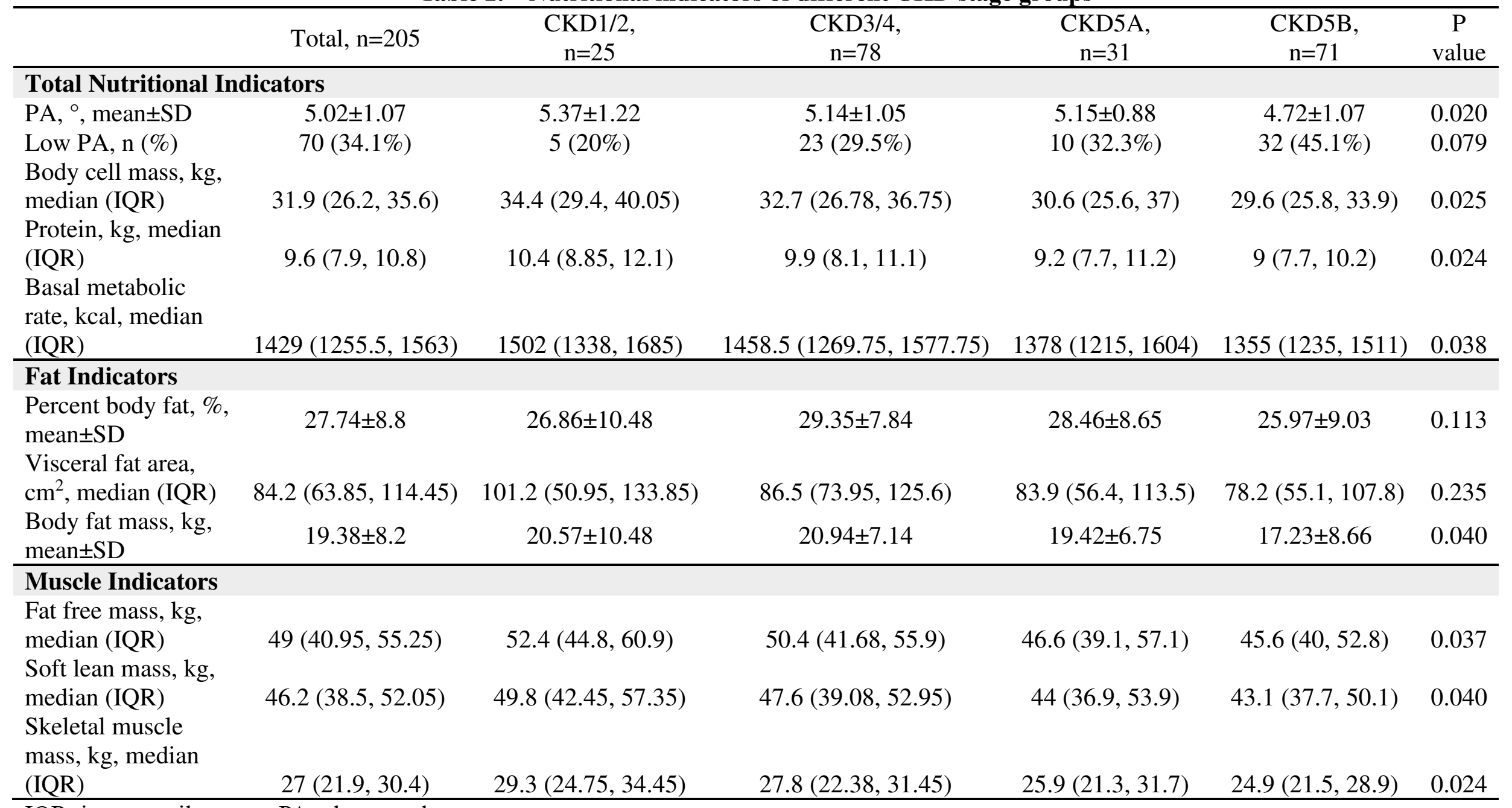

IQR, interquartile range; PA, phase angle. 


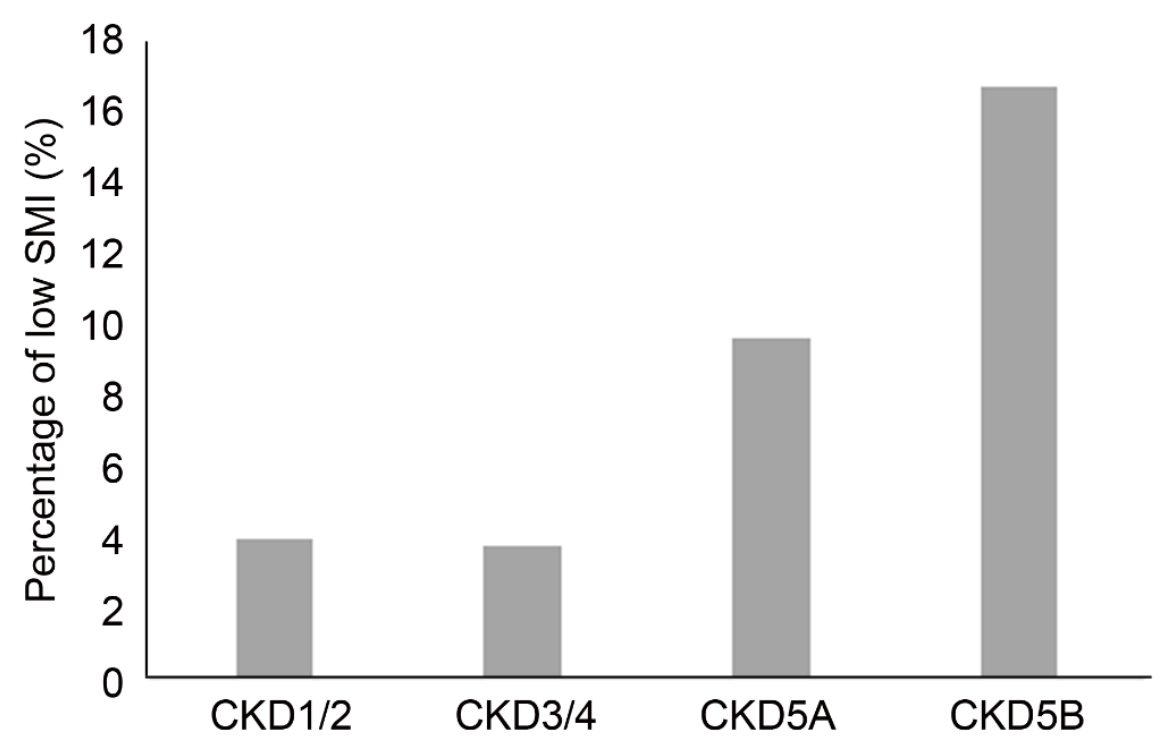

Figure 1. Percentage of low SMI at different CKD stage groups. (P value=0.037) 
Table 3. Correlation between InPTH and nutritional indicators

\begin{tabular}{ccc} 
& \multicolumn{2}{c}{$\operatorname{lnPTH}$} \\
\hline Variables & $\mathrm{r}$ & $\mathrm{p}$ value \\
\hline $\mathrm{BMI}$ & -0.14 & 0.043 \\
$\mathrm{Hb}$ & -0.14 & 0.046 \\
$\ln \mathrm{Alb}$ & 0.18 & 0.009 \\
$\operatorname{lnTc}$ & -0.22 & 0.001 \\
$\operatorname{lnTg}$ & -0.19 & 0.006 \\
$\mathrm{PA}$ & -0.09 & 0.220 \\
$\operatorname{lnBMR}$ & -0.11 & 0.117 \\
$\mathrm{PBF}$ & -0.11 & 0.105 \\
SMI & -0.14 & 0.043
\end{tabular}

PTH, parathyroid hormone; Hb, hemoglobin; Alb, albumin; Tc, total cholesterol; Tg, triglyceride; PA, phase angle; BMR, basal metabolic rate; PBF, percent body fat; SMI, skeletal muscle index. 
Table 4. Comparison between low and normal SMI groups

\begin{tabular}{|c|c|c|c|}
\hline Variables & Low SMI, $\mathrm{n}=19$ & Normal SMI, $\mathrm{n}=186$ & P Value \\
\hline \multicolumn{4}{|l|}{ Basic Indicators } \\
\hline Male, $\mathrm{n}(\%)$ & $3(15.8 \%)$ & $124(66.7 \%)$ & $<0.001$ \\
\hline CKD etiology, $\mathrm{n}(\%)$ & & & 0.080 \\
\hline Diabetic nephropathy & $3(15.8 \%)$ & $60(32.3 \%)$ & \\
\hline Hypertensive nephropathy & $1(5.3 \%)$ & $27(14.5 \%)$ & \\
\hline Unknown & $8(42.1 \%)$ & $33(17.7 \%)$ & \\
\hline CKD stage, n (\%) & & & 0.037 \\
\hline $1+2$ & $1(5.3 \%)$ & $24(12.9 \%)$ & \\
\hline $3+4$ & $3(15.8 \%)$ & $75(40.3 \%)$ & \\
\hline $5 \mathrm{~A}$ & $3(15.8 \%)$ & $28(15.1 \%)$ & \\
\hline $5 \mathrm{~B}$ & $12(63.2 \%)$ & $59(31.7 \%)$ & \\
\hline Total cholesterol, $\mathrm{mmol} / \mathrm{L}$, median (IQR) & $4.26(3.55,5.14)$ & $4.14(3.35,4.84)$ & 0.418 \\
\hline Triglyceride, mmol/L, median (IQR) & $1.46(0.99,2.22)$ & $1.71(1.17,2.44)$ & 0.626 \\
\hline Phosphorus, mmol/L, median (IQR) & $1.28(1.08,1.94)$ & $1.36(1.12,1.86)$ & 0.863 \\
\hline Calcium, mmol/L, median (IQR) & $2.19(2.12,2.55)$ & $2.18(2.07,2.35)$ & 0.326 \\
\hline \multicolumn{4}{|l|}{ Total Nutrition Indicators } \\
\hline $\mathrm{PA},{ }^{\circ}$, median $(\mathrm{IQR})$ & $4.2(3.4,4.7)$ & $5.2(4.38,5.9)$ & $<0.001$ \\
\hline Low PA, n (\%) & $14(73.7 \%)$ & $56(30.1 \%)$ & $<0.001$ \\
\hline Body cell mass, $\mathrm{kg}$, mean $\pm \mathrm{SD}$ & $22.43 \pm 1.76$ & $32.76 \pm 6.61$ & $<0.001$ \\
\hline
\end{tabular}


Protein, kg, median (IQR)

$6.9(6.3,7.1)$

$1134(1086,1177)$

$9.85(8.2,10.95)$

$1463(1285.75,1575.75)$

$<0.001$

Basal metabolic rate, kcal, median (IQR)

$28.37 \pm 10.12$

$68.7(49,122.1)$

$27.68 \pm 8.68$

$<0.001$

Percent body fat, $\%$, mean \pm SD

Visceral fat area, $\mathrm{cm}^{2}$, median (IQR)

$14.93 \pm 7.69$

$84.5(67.7,114.18)$

0.745

Body fat mass, $\mathrm{kg}$, mean \pm SD

$19.83 \pm 8.14$

0.105

Muscle Indicators

Fat free mass, kg, median (IQR)

Soft lean mass, kg, median (IQR)

Skeletal muscle mass, $\mathrm{kg}$, median (IQR)

$35.4(33.2,37.4)$

$33.1(31.2,35)$

SMI, $\mathrm{kg} / \mathrm{m}^{2}$, median (IQR)

$18.6(17.1,19.6)$

$50.6(42.4,55.83)$
$47.85(39.98,52.65)$
$27.6(22.8,31.18)$
$7.6(6.7,8.3)$

$<0.001$

$5.4(5,5.6)$

$<0.001$

IQR, interquartile range; PA, phase angle; SMI, skeletal muscle mass. 
Table 5. Relationship between PTH and low SMI (Binary logistic regression analysis)

\begin{tabular}{|c|c|c|c|c|c|c|}
\hline & \multicolumn{3}{|c|}{ Model 1} & \multicolumn{3}{|c|}{ Model 2} \\
\hline & OR $(95 \% \mathrm{CI})$ & $\mathrm{p}$ & C statistic & OR $(95 \% \mathrm{CI})$ & $\mathrm{p}$ & C statistic \\
\hline $\begin{array}{c}\mathrm{PTH} \leq 88 \mathrm{pg} / \mathrm{ml} \\
\mathrm{PTH}>88 \mathrm{pg} / \mathrm{ml}\end{array}$ & $\begin{array}{c}0.238(0.067-0.844) \\
4.205(1.185-14.924)\end{array}$ & 0.026 & 0.641 & $\begin{array}{c}0.085(0.008-0.928) \\
11.769(1.078-128.536)\end{array}$ & 0.043 & 0.986 \\
\hline
\end{tabular}

Model 1: Unadjusted;

Model 2: Adjustment factors were gender, age, BMI, hemoglobin, serum calcium, serum phosphorus, serum total cholesterol, serum triglyceride, and basal metabolic rate. 
Figures

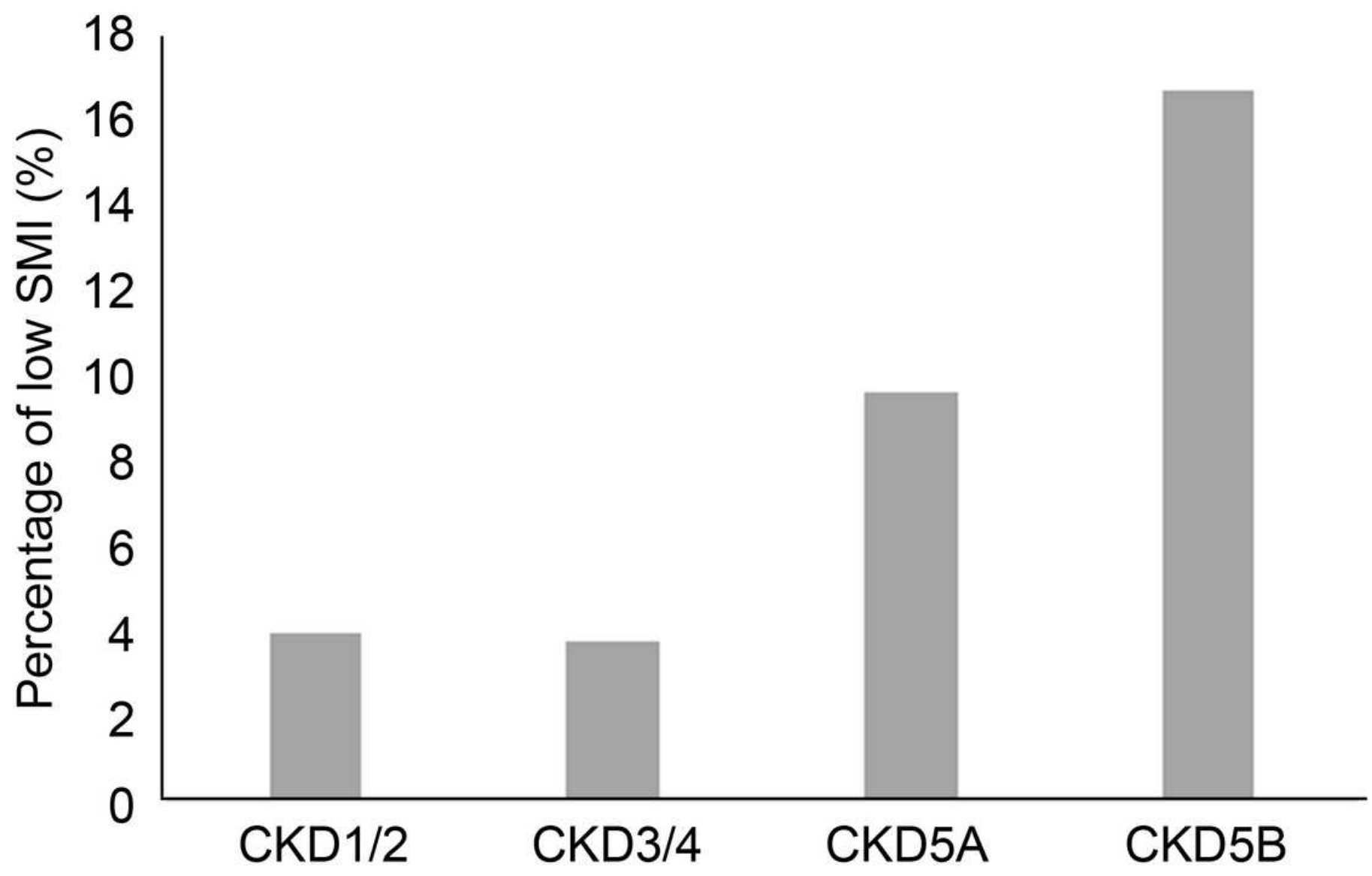

Figure 1

Percentage of low SMI at different CKD stage groups. (P value $=0.037$ )

\section{Supplementary Files}

This is a list of supplementary files associated with this preprint. Click to download.

- Table1.pdf

- Table2.pdf

- Table3.pdf

- Table4.pdf

- Table5.pdf 